\title{
Imaging in 3D under pressure: a decade of high-pressure X-ray microtomography development at GSECARS
}

\author{
Tony Yu*, Yanbin Wang and Mark L. Rivers
}

\begin{abstract}
The high-pressure X-ray microtomography (HPXMT) apparatus has been operating at the GeoSoilEnviroCARS (GSECARS) bending magnet beamline at the Advanced Photon Source since 2005. By combining the powerful synchrotron X-ray source and fast switching between white (for $X$-ray diffraction) and monochromatic (for absorption imaging) modes, this technique provides the high-pressure community with a unique opportunity to image the three-dimensional volume, texture, and microstructure of materials under high pressure and temperature. The ability to shear the sample with unlimited strain by twisting the two opposed anvils in the apparatus allows shear deformation studies under extreme pressure and temperature to be performed. HPXMT is a powerful tool for studying the physical properties of both crystalline and non-crystalline materials under high pressure and high temperature. Over the past 10 years, continuous effort has been put into technical development, modifications to improve the overall performance, and additional probing techniques to meet users' needs. Here, we present an up-to-date report on the HPXMT system, a brief review of some of its many exciting scientific applications, and a discussion of future developments.
\end{abstract}

Keywords: High pressure, Tomography, Synchrotron, Density, Rotation, Shear, Non-crystalline, Elasticity

\section{Review}

\section{Introduction}

Advances in high-pressure science rely critically on continuous technical developments and breakthroughs for new probes to characterize samples in situ under extreme conditions. With each new probing technique added to the tool kit, high-pressure research expands into new areas. Researchers have embraced every emerging new technique and have eagerly applied the techniques to scientific problems beyond the imagination of the original inventors. Literature shows that this has occurred throughout the history of high-pressure science. Excellent reviews highlighting the importance of new technical breakthroughs can be found in Bassett (2009) for the diamond anvil cell (DAC) and Liebermann (2011) for the multi-anvil press.

The development of three-dimensional (3D) tomographic imaging under high pressure and temperature

\footnotetext{
* Correspondence: tyu@cars.uchicago.edu

GeoSoilEnviroCARS, Center for Advanced Radiation Sources, The University of Chicago, 5640 S. Ellis Avenue, Chicago, IL 60637, USA
}

began at the turn of the 21st Century at GeoSoilEnviroCARS (GSECARS) of the Advanced Photon Source (APS; Argonne National Laboratory, IL, USA). In 2005, a highpressure X-ray microtomography apparatus was commissioned (Wang et al. 2005). The 3D tomographic imaging capability based on X-ray absorption was a major step toward investigating complex materials under high pressure and temperature. Soon after the commissioning of highpressure X-ray microtomography (HPXMT) at GSECARS, high-pressure tomography began at The European Synchrotron Radiation Facility (Bromiley et al. 2009) and Super Photon Ring-8 GeV (SPring-8) (Urakawa et al. 2010) for a large-volume press and at APS for the DAC (Liu et al. 2008). Since then, high-pressure imaging techniques have expanded from absorption tomography to diffraction (Alvarez-Murga et al. 2011) and beyond.

Since the inception of HPXMT apparatus, numerous developments have been made at GSECARS to improve the mechanical performance of the apparatus, expand the pressure and temperature capabilities, increase imaging quality, and reduce data collection time. This 
paper reviews major technical improvements made over the past decade to the HPXMT apparatus, highlights the scientific-rich applications, and discusses possible new directions for future developments.

\section{Basic setup of absorption-based microtomography}

The physical principle of absorption-based imaging is the Beer-Lambert Law, according to which the contrast of the X-ray image is a final result of the differences in density, mass absorption coefficient, and thickness of the objects through which the beam passes. A typical parallel beam full-field X-ray absorption imaging setup at the synchrotron light source consists of a fluorescent screen, an optical mirror, a long working distance focusing objective lens, and a charge-coupled device (CCD) or complementary metal-oxide-semiconductor (CMOS) camera (Fig. 1). A monochromatic X-ray beam with the desired photon energy (depending on the thickness, composition, and density of the object to be studied) passes through the sample and impinges on the fluorescent screen, which converts the X-ray radiograph into an image at a visible light wavelength. The visible light intensities in the visible image are linearly proportional to those of the transmitted X-rays. The visible image is then reflected by the mirror into the objective lens and captured by the camera. X-ray computed microtomography is performed by collecting a series of the abovementioned two-dimensional radiographs while rotating the sample along the axis perpendicular to the incident beam. The sample is rotated $180^{\circ}$ at a certain angular interval determined by the targeted linear resolution. After processing the absorption images using a filtered back-projection or similar algorithm to reconstruct the horizontal slice, a 3D reconstruction of the whole

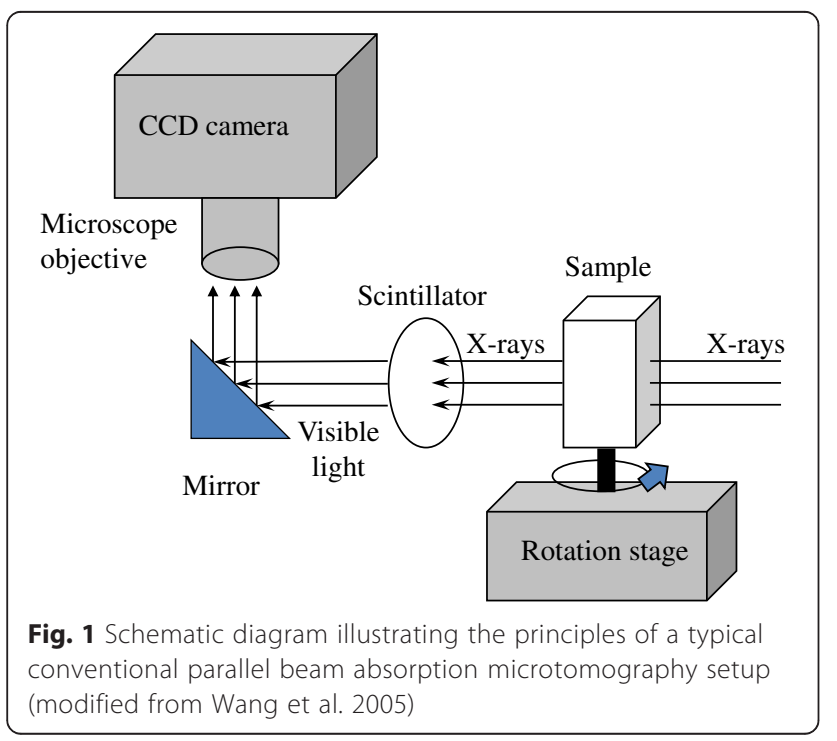

sample image is completed (Rivers et al. 1999, 2010). A tutorial on the tomography data processing procedure and software tools developed at GSECARS is available online (Rivers 1998).

\section{Pressure, temperature, and strain environment in the HPXMT apparatus Improving load capacity of the apparatus}

Figure 2a shows a cross section of the HPXMT apparatus. Anvils (A) are attached to the load supporting column (C), which is driven by low-profile, high-torque HarmonicDrive ${ }^{\mathrm{max}}$ units (B). The ends of column $\mathrm{C}$ are attached to spacers (D), which spread the load to a larger area and are supported by concentric needle rollers (E) mounted on steel plates (F). These steel plates are also part of a precision die-set, which allows the upper anvil $\left(A_{1}\right)$, load column $\left(C_{1}\right)$, and spacer $\left(D_{1}\right)$ to be lifted for sample loading and unloading (for details, see Wang et al. 2005).

The load supporting columns were originally straight cylinders, the diameter of which expanded under high load, causing them to bind to the HarmonicDrive ${ }^{\text {Tm }}$ fitting units from time to time. Frequent servicing was required to clean the interfaces and replace lubricant. Therefore, a modification was made to the columns (Fig. 2b, c). The new columns have a slightly smaller general diameter, with only three circular lines matching the inner diameter of the HarmonicDrive ${ }^{\mathrm{mm}}$ fitting units, to ensure axial alignment of the system. This has completely eliminated potential binding between the columns and the fitting units. In addition, two circular grooves were added to the column profile to alleviate potential gumming when lubricant hardens. After this modification, the system has been used routinely up to $50 \mathrm{t}$ (the designed load limit) and we have not seen any binding since. The mechanical strength and stability of the entire system have also been enhanced.

\section{Pushing pressure capability}

The pressure generating mechanism of the HPXMT apparatus is based on an opposed anvil design because of the need to have complete open access in the plane perpendicular to the vertical loading axis. Either Drickamer (Balchan and Drickamer 1961) or toroidal (Khvostantsev et al. 1977; Morard et al. 2007) devices can fulfill this requirement. The two anvils are pressed toward each other along the uniaxial load direction, with a sample assembly in between. Loading is provided by the $250 \mathrm{t}$ hydraulic press installed at 13-BM-D of the GSECARS sector. This press is used for a number of high-pressure devices, such as the deformation DIA and the T-cup apparatus. Each device is mounted in a designated die-set unit with wheels fitted in the rail system mounted on the press 

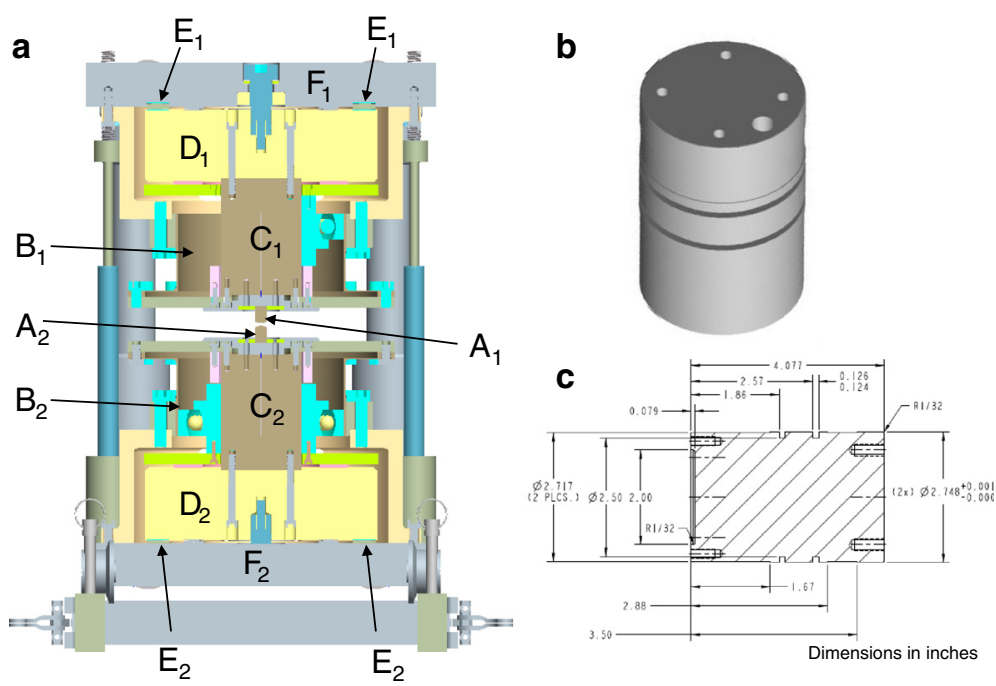

Fig. 2 Modification of the load-supporting column in the HPXMT apparatus. a Cross-sectional view of the apparatus. A anvil, $B$ HarmonicDrive ${ }^{\mathrm{TM}}$, $C$ load-supporting column, D load distribution spacer, $E$ needle rollers, $F$ base plate. Subscripts 1 and 2 denote upper and lower components, respectively. In the original design, $\mathbf{C}$ was a straight cylinder. $\mathbf{b}$ Modified load-supporting column $\mathbf{C}$. Note the grooves and depressions in the profile. c Cross section of modified column C

frame (Wang et al. 2009). This allows easy switchover between different devices to suit users' needs.

The Drickamer anvils are normally used with a containment ring to limit extrusion of the pressure medium under load. Figure 3 shows two typical cell assemblies along with two Drickamer anvil geometries (tapered vs. flat) used in HPXMT experiments. Two types of materials are used as containment rings, both are sufficiently X-ray transparent for imaging purposes.
An aluminum alloy sleeve has better mechanical strength to withstand high loads as well as high temperatures up to $1300 \mathrm{~K}$. At higher temperatures, both pressure media and containment ring become soft and can no longer support the uniaxial load, resulting in significant flattening of the sample. For relatively lowpressure and low-temperature $(<500 \mathrm{~K})$ experiments, a polyetherimide $\left(\mathrm{ULTEM}^{\mathrm{rm}}-1000\right)$ plastic sleeve is used for better X-ray transparency.
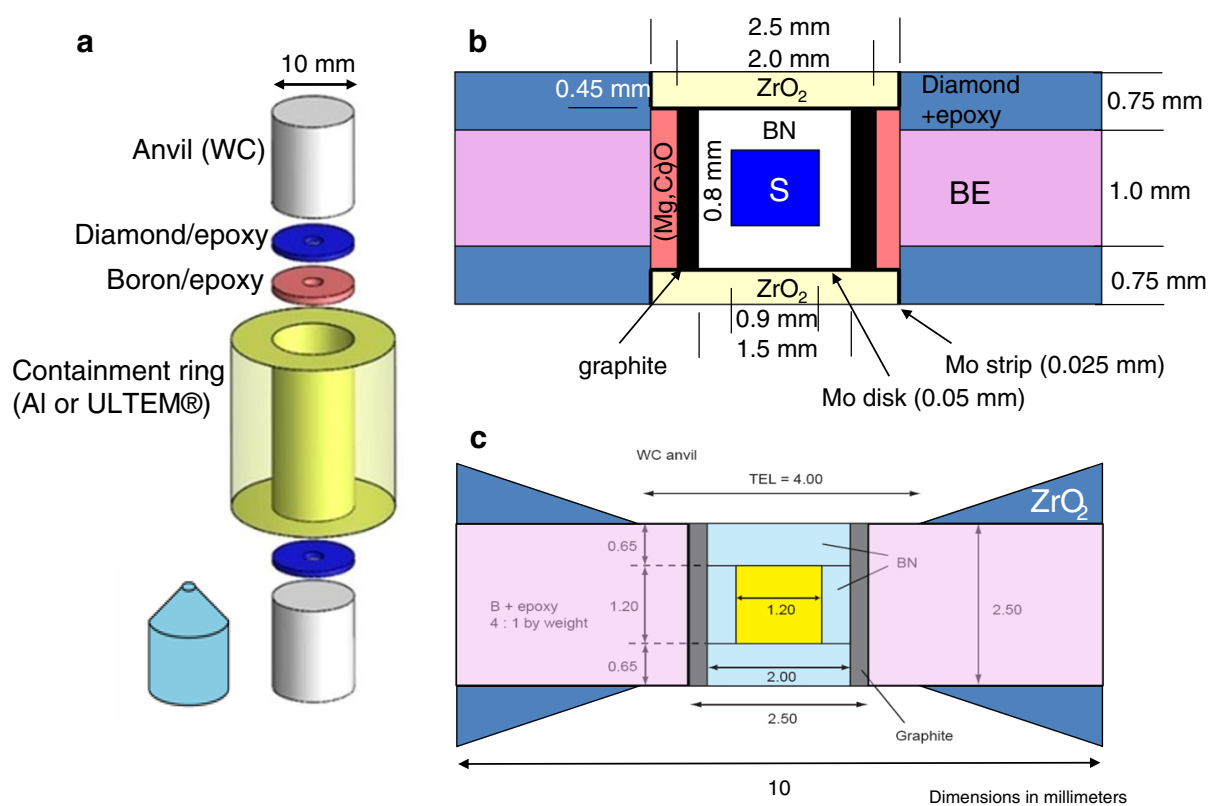

Fig. 3 Schematic illustration of the standard Drickamer cell with detailed cell designs. a Overall configuration. Both flat-top anvils and tapered anvils can be used. b Standard cell assembly for flat-top anvils. c Standard cell assembly for tapered anvils with 4 mm truncation 
The use of Paris-Edinburgh (PE)/toroidal anvils eliminates the need for a containment ring, which tends to attenuate absorption contrast. Extrusion of the pressure media is minimized by optimizing the anvil gap and gasket material outside the cell assembly (Kono et al. 2011). The anvil geometry and gasket material play an important role in pressure generation. Figure 4 shows a typical $\mathrm{PE}$ anvil geometry and the associated cell assembly. The depression in the PE/toroidal anvils allows a larger sample volume to be compressed with smaller thermal gradients than those in the Drickamer cell. However, a significant portion of the sample is hidden in the anvil depression, limiting imaging access. In addition, a small anvil gap and large gasket extrusion tend to lower the pressure generation efficiency.

Compared with the thick ( $50 \mathrm{~mm}$ wall thickness), strong (typically tungsten carbide, WC) conventional Drickamer containment rings, our containment rings are much thinner ( $5 \mathrm{~mm}$ wall thickness) and the materials are much weaker. This limits the maximum load of the system and hence the pressure capability. PE/toroidal anvils, on the other hand, require higher loads because of low pressure efficiency. Thus, in general, the pressure range in the HPXMT apparatus is limited to about $10 \mathrm{GPa}$ for Drickamer cells and $\sim 5 \mathrm{GPa}$ in PE/toroidal cells. A new anvil design was developed by Kono et al. (2014), which combines the favorable features of both

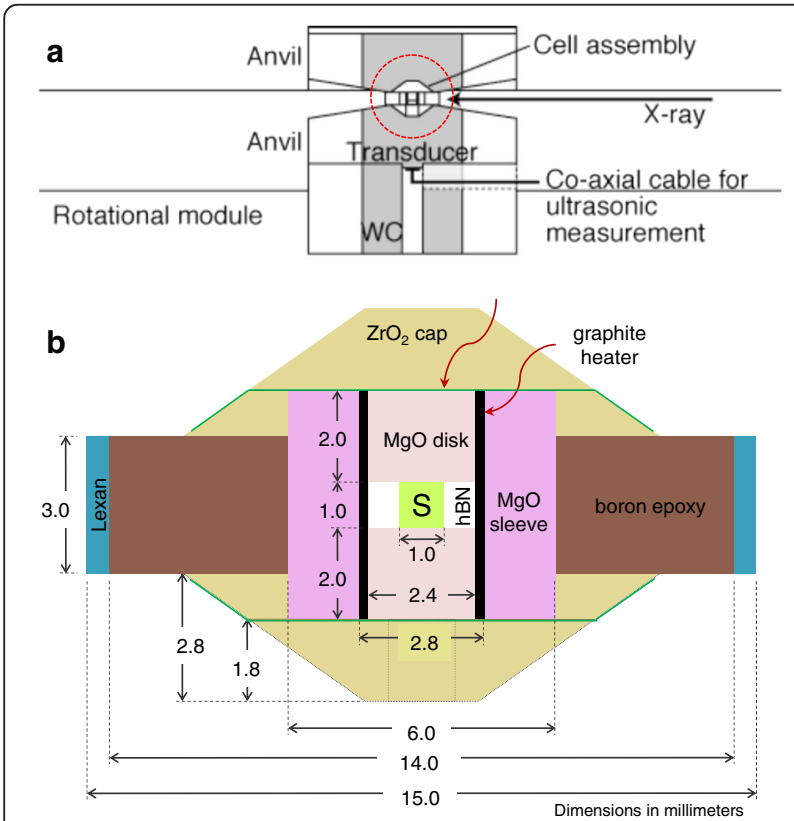

Fig. 4 Schematic diagram of the PE cell with details of the cell design. a Configuration of the PE anvils. WC anvils (gray) are $25 \mathrm{~mm}$ in diameter, with steel rings outside the anvils. An ultrasonic transducer can be attached to the bottom of the lower anvil for acoustic velocity measurements. b Standard cell assembly (red dotted circle in a) the Drickamer and toroidal anvils. This cupped Drickamer/toroidal (CDT) anvil has a flat anvil top (unlike tapered PE/toroidal anvils) similar to the Drickamer design, but with a ring-shaped groove and a central depression similar to the PE/toroidal anvils (Fig. 5). This has significantly expanded our pressure and temperature capabilities up to $12 \mathrm{GPa}$ and $2000 \mathrm{~K}$, respectively. With future development, the setup could potentially reach a pressure of $20 \mathrm{GPa}$.

\section{Temperature generation}

We use conventional resistive heating to generate high temperatures with an AC power supply. Graphite is generally used as the heating material because of its excellent X-ray transparency. A graphite heater can steadily provide high temperatures up to $2000 \mathrm{~K}$. For experienced users, $2200 \mathrm{~K}$ can be reached routinely. However, with the pressure capability above $10 \mathrm{GPa}$, graphite can no longer be used, because of the formation of diamond. We have tested $\mathrm{TiB}_{2}$ as a highpressure heater material from 5 to $45 \mathrm{t}$ (Fig. 6a). The results show that the $\mathrm{TiB}_{2}$ heater is capable of generating stable temperatures up to $2000 \mathrm{~K}$. A slight drawback is that during heating, the electrical resistance does not vary linearly (Fig. 6b). Peak values and temperatures of the resistance turnover are probably due to a phase transition in $\mathrm{TiB}_{2}$, as the resistance after the turnover follows a smooth curve (Fig. 6b).

In HPXMT experiments, the temperature is estimated based on the temperature-power relation, which was established in separate experiments using a thermocouple.

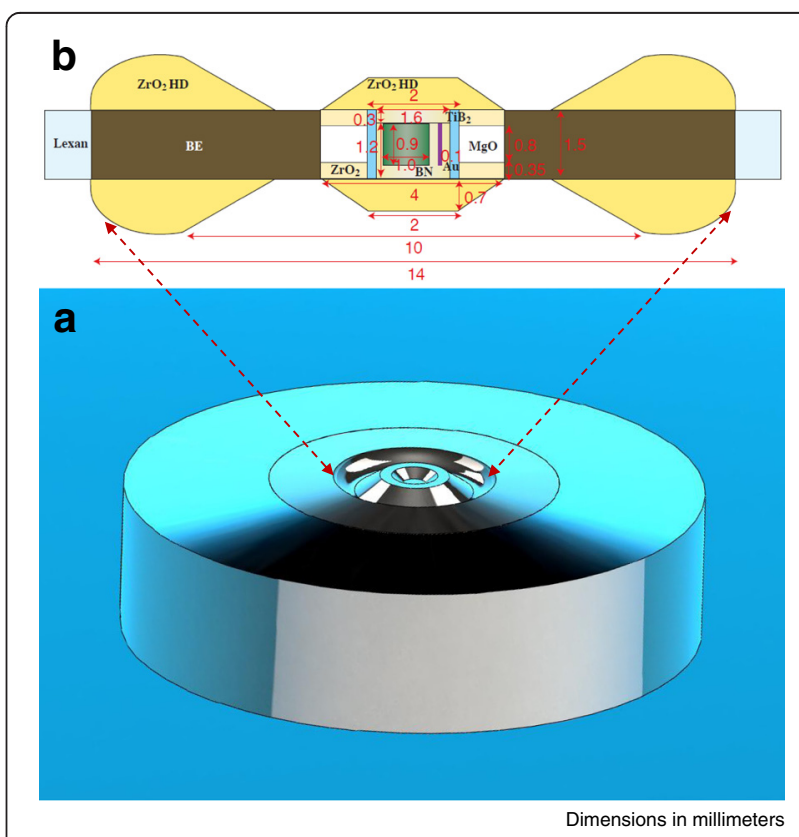

Fig. 5 CDT anvil with detailed cell design. a Illustration of the general shape of the CDT anvil. b Cell assembly for the CDT anvil cell 


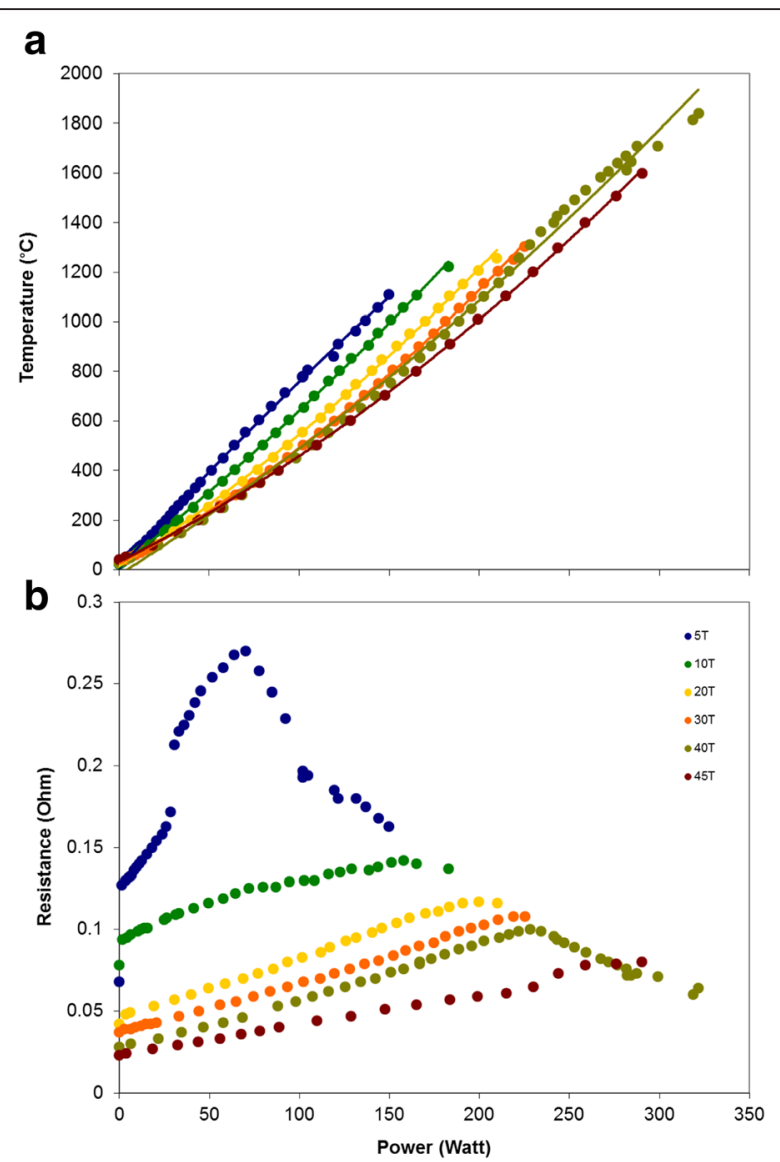

Fig. 6 Performance of $\mathrm{TiB}_{2}$ heater in the CDT cell. a Temperature vs. power curves as measured by using a thermocouple in the test cell. Note that the temperature-power relation varies with applied load. b Resistance of the $\mathrm{TiB}_{2}$ heater with power consumption at various ram loads. The complex relation requires heating to be conducted slowly

We find that the temperature-power relations vary with applied ram load, possibly reflecting deformation in the heater as well as changes in the distance between the heater and the anvil tips (Fig. 6a). This introduces some uncertainties in temperature measurements. A series of calibration curves is needed to obtain reliable temperature estimates. Alternatively, several metal foils ( $\mathrm{Ag}, \mathrm{Au}$, etc.) with well-known melting curves can be placed in a single cell with in situ imaging to detect the onset of melting by monitoring the shape change in the foils, providing temperature fixed points (Clark et al. 2013).

\section{Controlled deformation in HPXMT apparatus}

In addition to quasi-hydrostatic experiments, two types of deformation experiments have been performed using the HPXMT setup to visualize microstructural evolution. One is uniaxial compression through the top and bottom Drickamer anvils. With a containment ring that is mechanically stronger than the sample, the sample deforms under uniaxial load (Fig. 7). This technique works best for porous or highly heterogeneous materials where the pressure is ill-defined at microscopic scales (e.g., Sanematsu et al. 2015). The second type of deformation is shearing the sample in the horizontal plane under compression. The HPXMT apparatus uses two independent driving trains to rotate the upper and lower anvils (e.g., Wang et al. 2011). Twisting the two anvils in opposite directions generates essentially unlimited shear strain in the sample. In order to reach sufficient mechanical coupling between the anvils and the sample interfaces, uniaxial compression is needed. Tapered anvils are often used to achieve higher pressures, whereas in the case of flat anvils, better mechanical coupling between the anvils and the sample interfaces is achieved. Although there may be slippage between the anvil-cell and pressure medium-sample interfaces, 3D microstructural information contained in tomographic images is ideal for characterizing true deformation in the sample (e.g., Wang et al. 2005; Todd 2013).

\section{System setup and upgrades Imaging setup and upgrades}

The HPXMT setup utilizes the synchrotron bending magnet beam (13-BM-D) at the APS. A Si(111) monochromator is used to select monochromatic radiation from 7 to $65 \mathrm{keV}$. Typical photon energies used for the HPXMT apparatus range from 25 to $45 \mathrm{keV}$. Initially, we used yttrium aluminum garnet (YAG) as scintillator crystals $(0.3 \mathrm{~mm}$ thickness). This is adequate for lower energies, but at higher energies, the beam penetration is deeper in the scintillator. The depth of focus of the objective lens cannot discriminate signals from different depths of the scintillator, resulting in poorer resolution. Switching to $\mathrm{CdWO}_{4}$ scintillators improved image sharpness somewhat. Currently, we use single-crystal cerium-doped lutetium aluminum garnet $\left(\mathrm{Lu}_{3} \mathrm{Al}_{5} \mathrm{O}_{12}\right)$ or Ce:LuAG (0.1 mm thickness). Because Lu has a much higher atomic number than Y, this screen has a higher stopping power. Rivers et al. (2010) have evaluated and compared the image quality between the YAG and Ce:LuAG scintillator crystals and shown that the image generated by the Ce:LuAG crystal is notably sharper.

Our imaging cameras have gone through several generations. Currently, we use Point Grey CMOS Model GS3-U3-23S6M, which has a $1920 \times 1200$ pixel array with a dynamic range of $73 \mathrm{~dB}$. It has a maximum speed of 162 frames per second with a peak quantum efficiency of $76 \%$.

\section{New integrated press, detector, and imaging position system}

An integrated control and position system was installed in 2012 for more precise control with maximum flexibility for the $250 \mathrm{t}$ press as well as diffraction detectors. In 

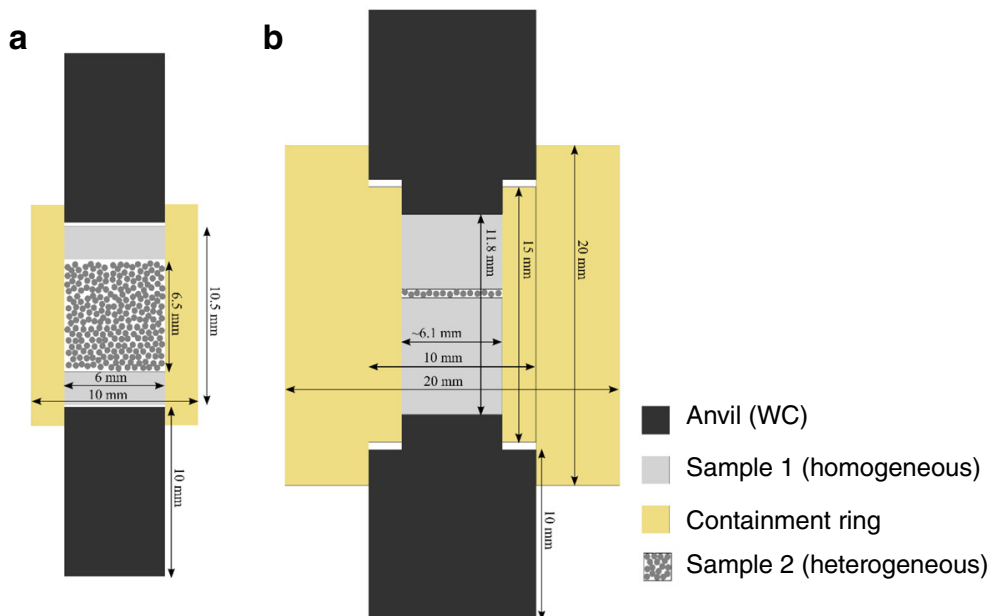

Fig. 7 Uniaxial load cells for low-pressure compression experiments. A bulk-proppant (a) and a monolayer-proppant (b) experimental setups are shown (modified after Sanematsu et al. 2015)

the new system, press positioning and detector positioning are designed as two separate subsystems, both of which share a set of guide rails mounted to a large base plate that is grouted to the hutch floor. This allows for manual adjustment of the distance between the press and the detector. The press positioning system has motorized and encoded motions with three linear translations and one rotation (manual) with respect to the vertical loading axis. The detector positioning system consists of five motorized and encoded motions: three linear translations and two rotation axes. Figure 8 illustrates the entire nine-axis system (Deyhim et al. 2012). Linear position accuracy has improved to within $0.001 \mathrm{~mm}$, and rotations of the detector support are within $0.002^{\circ}$.

A narrow frame has been added between the press and detector manipulation systems for the imaging camera and scintillator assembly. This provides a more stable support for the imaging system and allows

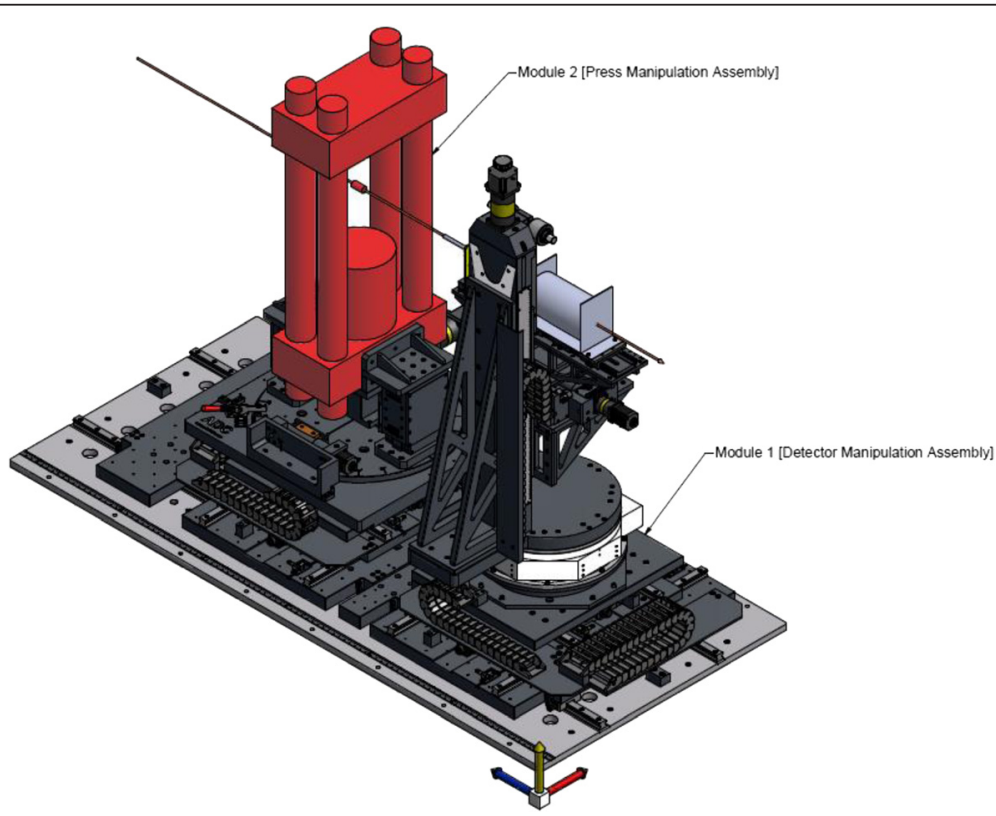

Fig. 8 Upgraded control system for the $250 \mathrm{t}$ press and detector support in 13-BM-D. The five-axis detector manipulation subsystem (module 1) and the four-axis press subsystem (module 2) are aligned on a same pair of rails. This allows the sample-detector distance to be varied as needed. A narrow frame was added between the two subsystems (not shown), for supporting the imaging setup (phosphor assembly, imaging camera) and ion chamber, etc., as well as their linear motion control components 
sample-scintillator distance to be freely adjusted, making it possible to apply phase contrast imaging technique to the HPXMT setup in the future.

The load feedback function in the $250 \mathrm{t}$ press is less favorable during high-temperature data collection; the sample height may change because of the uniaxial nature of the opposed anvil devices, as the hydraulic ram compresses the HPXMT module along the vertical axis. This results in blurry tomography images. We have implemented a precision linear-voltage displacement transducer and added an option for feedback on the position of the hydraulic ram cylinder. This option keeps the sample cell vertical movement to a minimum throughout the tomography data collection.

\section{Integrating additional probe techniques}

Ultrasonic elastic wave velocity measurement has been implemented in the HPXMT setup (Kono et al. 2011). Figure $4 \mathrm{a}$ shows the location of the acoustic transducer for acoustic travel time measurement. Sample length is conveniently measured by $\mathrm{X}$-ray imaging. This is extremely useful especially for studying non-crystalline materials.

\section{Data collection, processing, and image reconstruction New on-the-fly data collection mode}

In the past, the collection was done in a stop-and-scan mode, with the rotation starting and stopping at each angle. The overhead was typically about $0.2-0.3 \mathrm{~s}$ at each point, depending on the motor velocity and acceleration values used. Not only did it increase the total data collection time, but also the frequent abrupt start and stop motion was also unfavorable for the mechanics of the HPXMT apparatus, especially when the system was under high load. To eliminate the start/stop overhead, Rivers et al. (2010) implemented on-the-fly data collection, with continuous motion of the rotation stage. Now, the motor pulses from the stepper motor controller are fed into an SIS3820 multichannel scalar, which divides the motor pulses by the number of motor pulses per rotation angle increment $(N)$. The speed of the rotation stage is set so that the stage moves by 1 angular step (e.g., $0.25^{\circ}$ ) in the time required for the desired exposure plus the detector readout. If the optimum number of projections $\left[(\pi / 2) \times N_{X}, N_{X}\right.$ is the number of horizontal pixels)] is acquired, then the outermost part of the sample moves less than 1 pixel during the time to collect one radiograph in this continuous motion mode. This development has greatly improved the mechanical stability of the HPXMT system.

\section{Dark current and flat field normalization}

The raw data collected with the Point Grey camera are 12 bit $1920 \times 1200$ pixel images. The first correction that must be applied to such images is for dark current and flat field. Dark current is the signal recorded in the absence of X-rays. We typically take multiple $5 \mathrm{~s}$ exposures to obtain an average dark current. The flat field is the image that is measured with the $\mathrm{X}$-rays on, but without a sample in the beam. The non-uniformity in the flat field includes effects of non-uniformities in the incident $\mathrm{X}$-ray beam and non-uniform responses of the scintillator and the imaging detector. In ambient tomography, the sample is surrounded by nothing except for air. In HPXMT, the sample is surrounded by a capsule, a heater, and layers of pressure medium, plus the containment ring. In order to maximize pixel coverage on the sample for better spatial resolution, we use the pressure medium as the intensity background instead of air. Flat field images are taken through a dummy cell assembly, which has identical pressure medium to the true sample assembly, but with the sample removed. In practice, the dummy is mounted $\sim 20 \mathrm{~mm}$ away from the highpressure assembly; flat field images are taken by driving the hydraulic press so that the dummy is in the X-ray path.

\section{Reconstruction}

The speed of image reconstruction process was significantly improved by using the new tomoRecon multi-threaded code (Rivers 2012). This code uses the high-speed Gridrec FFT algorithm and reconstructs $N$ slices in parallel on $N$ cores in the workstation. The time to reconstruct a 1920 $(\mathrm{X}) \times 1200(\mathrm{Y}) \times 900$ (projections) dataset is now less than $60 \mathrm{~s}$. The reconstruction code is written in $\mathrm{C}++$ as an IDL user interface. If needed, the reconstruction code can be run free of cost by users in their home institutions.

\section{Rotation center correction}

The high-pressure nature of HPXMT apparatus makes the rotation center mechanically less accurate than conventional tomography with high-precision motors. The poor centering causes artifacts in the tomography images. During data processing, the software determines the "center-of-gravity" of each row in each sinogram and fits this center-of-gravity array to a sine wave. This results in a sinogram which is centered better on the rotation axis. The sinogram is then shifted left or right so that the rotation axis is located on the center column of the sinogram array. In cases where this automatic centering function is insufficient for the correction, a manual tweak function is available to tweak the sinogram until the resulting reconstruction is satisfactory.

\section{Post analysis}

Image analysis and manipulation after tomography reconstruction are conducted using free software such as ImageJ (http://imagej.nih.gov/ij/) and Blob3D (Ketcham 2005; http://www.ctlab.geo.utexas.edu/software/blob3d/). 
The tool kits included in these software packages allow texture analyses, strain calculations, and volume calculations. Especially for ImageJ, numerous special tools have been created by its user community and many are available online free of charge. Sophisticated users use commercially available software packages that have the specific functions they need.

\section{Application highlights}

A wide range of scientific projects have used highpressure X-ray microtomography. Topics cover subjects in many fields such as physics, materials science, earth science, and civil engineering. In this section, we highlight recent applications that have made a significant contribution to the corresponding field.

\section{Density of non-crystalline material under pressure}

Measuring the density of non-crystalline materials, such as glasses and melts, under high pressure has been difficult. X-ray diffraction, the most convenient and commonly used technique for crystalline materials, does not directly yield density information for non-crystalline materials because they have no long-range periodic atomic arrangement. Independent measurements of the volumetric and elastic properties are required to determine the physical properties of non-crystalline materials at high pressures. HPXMT provides two approaches for density measurement: (1) direct volume rendering under high pressure and temperature and (2) determining the linear absorption coefficient $\left(\mu_{l}\right)$ in each voxel (voxel intensities in reconstructed tomographic images are directly proportional to $\mu_{l}$ ). In the first approach, determining the image threshold between the sample and the pressure medium is critical for accurate volume measurement (Lesher et al. 2009). By direct volume rendering, Lesher et al. (2009) investigated compression of glasses of several compositions in the $\mathrm{Mg}_{2} \mathrm{SiO}_{4}-\mathrm{MgSiO}_{3}$ pseudo-binary system up to $11.5 \mathrm{GPa}$ and determined bulk moduli (Fig. 9a, b). High-temperature measurements of $\mathrm{MgSiO}_{3}$ glass at $2 \mathrm{GPa}$ revealed negligible thermal expansion up to $\sim 750{ }^{\circ} \mathrm{C}$, which is close to the glass transition temperature at $1 \mathrm{~atm}\left(765^{\circ} \mathrm{C}\right.$; Wilding et al. 2004). Above $750{ }^{\circ} \mathrm{C}$, this glass exhibits a large volumetric thermal expansion $\left(21 \times 10^{-5} \mathrm{~K}^{-1}\right)$. In the second approach, density is obtained from $\mu_{l}$ through the relation $\mu_{l}=\mu_{m} \times \rho$ by assuming that the mass absorption coefficient $\left(\mu_{m}\right)$ of materials remains constant under modest pressure and temperature conditions (Lesher et al. 2009). Note, however, that voxel intensities may be skewed under high pressure by the presence of the pressure medium surrounding the sample, especially after applying non-conventional flat-field normalization. Therefore, it is desirable to establish a calibration

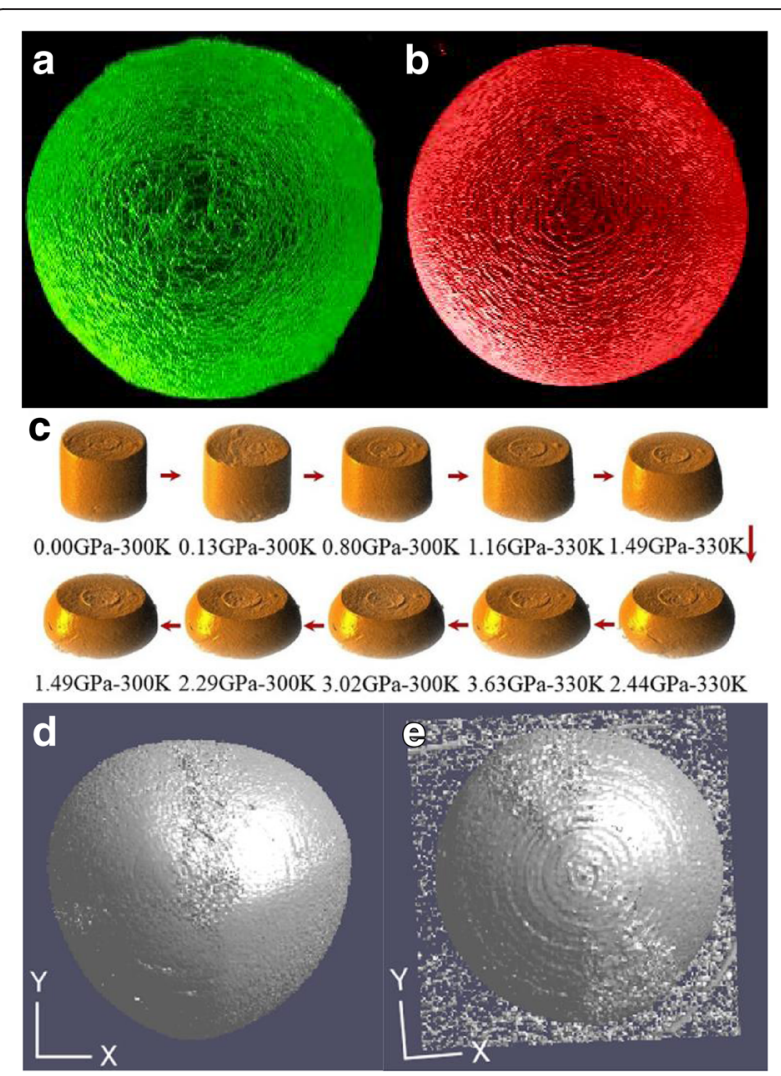

Fig. 9 Examples of volume rendering at high pressure and temperature by tomography reconstruction. A vitreous $\mathrm{Mg}_{2} \mathrm{SiO}_{4}$ sphere (initial diameter $=0.803 \mathrm{~mm}$ ) at $\mathbf{a} 1 \mathrm{~atm}$ and $\mathbf{b} 1 \mathrm{GPa}$. Images modified after Lesher et al. (2009). c A series of images of a Ga cylinder at various pressure and temperature conditions. Images modified after Li et al. (2014). Sessile drop of $\mathrm{Fe}_{60} \mathrm{~S}_{40}$ melt at $2 \mathrm{GPa}$ and $\mathbf{d} 1207$ and e $1293 \mathrm{~K}$. Note the semi-triangular shape of the drop at $1207 \mathrm{~K}$, indicating that the sample has not reached equilibrium with the surrounding sodium disilicate liquid. Therefore, cross-sectional measurements on this drop cannot give accurate interfacial energy. Images modified after Terasaki et al. (2008)

relation by including reference materials with known densities in the same cell assembly (Lesher et al. 2009).

Volumetric rendering was also applied to solid and liquid $\mathrm{Ga}$ to study its compression behavior ( $\mathrm{Li}$ et al. 2014). The rapid switching between monochromatic (for tomography imaging) and white mode (for energydispersive diffraction) made it convenient to identify crystalline phases and melting. This technique was described by Lesher et al. (2009). Two isothermal compression curves were obtained on liquid $\mathrm{Ga}$ at 300 and $330 \mathrm{~K}$ up to $3.6 \mathrm{GPa}$ (Fig. 9c). Above 2.44 GPa, the $330 \mathrm{~K}$ curve showed a significant bend-over, which was interpreted as due to a liquid-liquid transition.

\section{Measuring interfacial tension by imaging liquid droplets}

A novel application of the volume rendering measurement in HPXMT is to measure interfacial tension 
between liquids under high pressure and temperature. The fundamental principle is the well-known Laplace pressure caused by the surface tension or energy of the interface between liquid and gas. At 1 atm, a liquid $\left(\mathrm{L}_{1}\right)$ drop sitting on the flat surface of a solid in another liquid $\left(\mathrm{L}_{2}\right.$; or gas such as air) takes a certain shape, which is determined by the balance of the surface energies between $L_{1}$ and $L_{2}\left(\gamma_{L 1-L 2}\right)$, liquid and solid $\left(\gamma_{L 1-S}\right)$, and solid and air $\left(\gamma_{\mathrm{S}-\mathrm{L} 2}\right)$ in the presence of a density difference $(\Delta \rho)$ between $L_{1}$ and $L_{2}$. This balance is shown by the contact angle $\left(\theta_{\mathrm{C}}\right)$ between the drop of $\mathrm{L}$ and the solid plate. The technique based on this principle to measure interfacial tension is called the sessile drop method (Rotenberg et al. 1983), which shows that if the drop is axisymmetric (with the symmetry axis parallel to gravity), surface tension between liquids $\mathrm{L}_{1}$ and $\mathrm{L}_{2}$ can be determined by measuring the shape of the liquid drop. Terasaki et al. $(2008,2009)$ extended this technique to high pressures and measured the surface tension of liquid $\mathrm{Ni}-\mathrm{S}, \mathrm{Fe}-\mathrm{S}$, and $\mathrm{Fe}-\mathrm{P}$ in liquid sodium disilicate by using HPXMT. A fully densified alumina plate was used in the high-pressure cell assembly for the sessile drop. They found that at $1.5 \mathrm{GPa}$, the interfacial tension of liquid Fe-S decreased significantly from 802 to $112 \mathrm{mN} / \mathrm{m}$ with increasing sulfur content from 0 to 40 at.\%. This effect of 40 at.\% S on interfacial tension was large compared with the effect of temperature $(\sim 273 \mathrm{mN} / \mathrm{m}$ reduction with an increase of $200 \mathrm{~K})$. In contrast, interfacial tension in the Fe-P system increased only slightly from 802 to $873 \mathrm{mN} / \mathrm{m}$ as phosphorus content increased to 17 at.\%. Both tendencies are consistent with those measured at ambient pressure on $\mathrm{Fe}-\mathrm{S}$ and Fe-P liquids.

Only when the shape of the drop is axisymmetric can the sessile drop method be used for interfacial tension measurement. HPXMT is critical in checking the shape of the drop in situ at high pressure (Fig. 9d, e). When a melt is quenched, the shape of the drop changes suddenly, resulting in drastically different apparent interfacial tension values, if the quenched shape is used. Furthermore, it must be ensured that the melt is compositionally homogeneous. Because Fe and S (or P) have very different densities and mass absorption coefficients, any segregation of Fe and/or $\mathrm{S}(\mathrm{P})$ in the melt can be easily observed (Terasaki et al. 2009).

\section{D imaging of microstructural heterogeneities}

The power of HPXMT apparatus lies in the ability to resolve small heterogeneities under high pressure and temperature and various strain conditions. This allows in situ tracking of various components in complex materials, which is required in many scientific disciplines. Below are a few examples highlighting the great potential of this technique.
Shear-induced fabric transition in multiphase composites Textural evolution of two-phase composites (San Carlos olivine $+\sim 10$ vol.\% Fe-Ni-S) during large shear deformation was studied by applying the unique twisting capability of the HPXMT apparatus (Wang et al. 2011). In these composites, olivine is the strong phase in the rheological sense, whereas $\mathrm{Fe}-\mathrm{Ni}-\mathrm{S}$ is the weaker phase. Two samples with distinct textures were examined. In sample 1, round-shaped $\mathrm{Fe}-\mathrm{Ni}$-S inclusions dispersed in the olivine matrix, forming a load-bearing framework (LBF) texture. In other words, when such a sample is deformed, the phase that supports the applied stress is primarily olivine. Sample 2 consisted of an olivine matrix separated by interconnected channels of quenched Fe-Ni-S melt. The rheology of this sample would be dominated by the weaker Fe-Ni-S phase. Both samples were compressed to $6 \mathrm{GPa}$ and deformed at temperatures up to $800 \mathrm{~K}$. A series of 3D tomographic images was collected at various twist angles. Figure 10ad shows texture evolution in sample 1. Significant slippage was observed at the initial stage of the shear deformation process. By a total twisting angle of $50^{\circ}$, robust mechanical coupling was established, although slippage was never completely eliminated. Analysis of subsequent 3D images allowed systematic tracking of the shear deformation process and accurate measurement of the shear strain. Fe-Ni-S inclusions in sample 1 were carefully analyzed, tracking their aspect ratios and long-axis inclination angles with respect to the shearing plane with increasing shear strain. The initially equant $\mathrm{Fe}$ $\mathrm{Ni}-\mathrm{S}$ inclusions flattened and smeared along the shear plane, transforming gradually from LBF to the interconnected weak layer texture. During this texture transition, the rheological properties of the bulk sample changed from being primarily dominated by the stronger olivine phase to being dominated by the weaker Fe-Ni-S phase. A similar texture transition is expected to occur in the Earth's mantle, which consists of multiple rock-forming minerals, with significant geodynamic implications (Wang et al. 2011).

\section{Shear deformation in metal-silicate composites and applications to core formation processes in planetesi- mals Solid samples of San Carlos olivine + 4.5 vol.\% FeS were compressed in the HPXMT apparatus, heated, and then sheared by twisting the upper and lower Drickamer anvils, under the conditions (1.5 GPa and $1373 \mathrm{~K}$ ) where Fe-S was liquid and olivine remained solid. Each twisting step involved with a total $180^{\circ}$ relative rotation between the upper and lower anvils. After each twisting step, a 3D image of the sample was obtained after cooling to $1123 \mathrm{~K}$, to avoid unwanted motion of the FeS liquid pockets. The total angular rotation was $840^{\circ}$ with an estimated max- imum apparent shear strain rate of $7.7 \times 10^{-5} \mathrm{~s}^{-1}$. The resulting digital volumes were processed (Fig. 11), and}




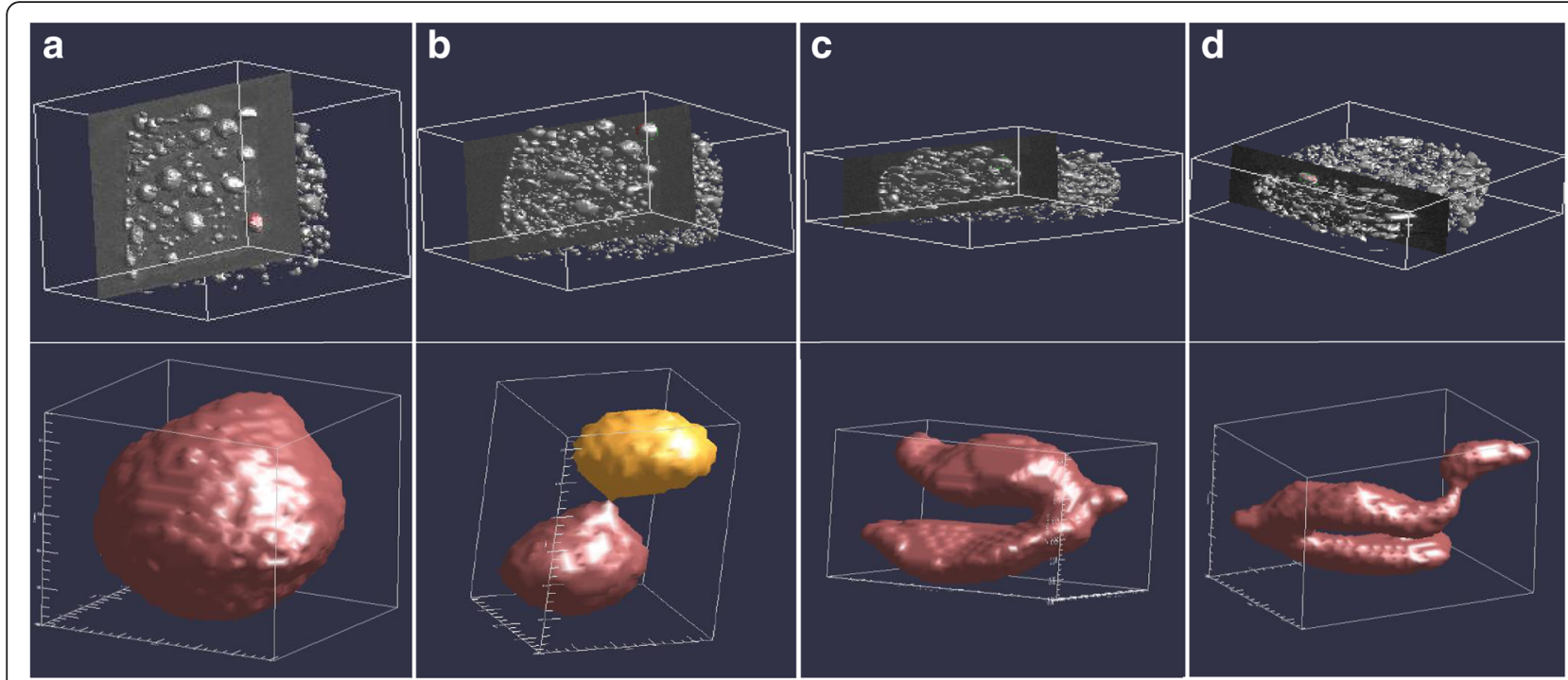

Fig. 10 High-pressure and high-temperature microstructural evolution of olivine and Fe-Ni-S composite sample under various shearing strains. In the sample images (San Carlos olivine +10 vol.\% Fe-Ni-S), the olivine matrix has been digitally removed, showing alloy blobs, the shape and spatial distribution of which change with shear strain. Images are presented as upper- and lower-panel pairs, with the upper panel showing the entire sample and the lower panel showing particular blob configurations. a Sample under ambient conditions. Blobs are separate and equant. b Sample at $6 \mathrm{GPa}$ and $800 \mathrm{~K}$, with no shear strain applied by anvil twisting. Blobs become more elliptical with some interconnection due to axial shortening. c Sample at $6 \mathrm{GPa}$ and $800 \mathrm{~K}$, upper and lower sample surface twisted by $30^{\circ}$ (as indicated by tracking individual blobs). Blobs are more flattened and elongated along the shear direction, and more blob connections are observed. $\mathbf{d}$ Sample at 6 GPa and 800 K, upper and lower sample surface twisted by $65^{\circ}$. Eventually, blobs become film-like and can no longer be separated. Modified after Wang et al. (2011)

permeability simulations were performed utilizing the lattice Boltzmann method (LBM) to determine the effect of shear deformation on connectivity and permeability within the sample. Using the average of the permeabilities in the shearing direction (about $6.3 \times 10^{-15} \mathrm{~m}^{2}$ ), the liquid FeS migration velocity was calculated to be $4 \mathrm{~mm} /$ year (Todd et al. 2016). This suggests that the migration velocity was not high enough for complete core formation to take place in planetesimals within the 1-5 My time frame, based on W-Hf isotope data (e.g., Kleine et al. 2009). The HPXMT technique in conjunction with LBM analysis appears promising; further work utilizing these methods will bring us closer to understanding the complex and significant event of core formation in planetesimals.

\section{Bulk-proppant packs and propped fractures under} uniaxial load The permeability of the reservoir is a major controlling factor in oil/gas production rates. In order to increase production rate in rock formations that are low in permeability (e.g., tight sands or shales), injecting proppants into the reservoir after rock fracturing processes has become a routine procedure in the field. Although this has been proven to be an effective way to increase production, there is still much about the method that we need to understand. What remains unclear is how the proppant-filled fracture system responds to the change in loading stress. For example, the impact of the stress change on the permeability, conductivity, and proppant structure and fracture width is not fully understood. Sanematsu et al. (2015) performed tomography experiments using the HPXMT setup to image two different unsaturated rock/fracture/ proppant systems, one with shale as the representative wall rock and the other with Berea sandstone. In each study, proppants were loaded between two layers of wall rock and the layers were stacked together along the uniaxial stress direction. Tomography images were collected for both systems at various stress levels typical of oil-producing reservoirs (Fig. 12a-e). Quantitative grain analysis based on the 3D images showed the expected changes as stress increased: rearrangement of the packing structure, a corresponding reduction in porosity, and some embedding at the rock walls. With increasing stress, image-based finite element method (FEM) and LBM simulations showed trends toward a more uniform velocity fields in both cases. This is due to elimination of the largest flow channel in the proppantsandstone setup. However, in the proppant-shale experiment, a more uniform velocity field was the result of narrowing of the main flow channels. Both FEM and LBM simulations predicted similar permeabilities within the applied stress range, and the permeability was essentially independent of loading at lower stresses (below $83 \mathrm{MPa}$ ). The HPXMT setup has proven to be a very powerful tool in providing information to help understand the proppants' reaction to uniaxial loading stresses. 


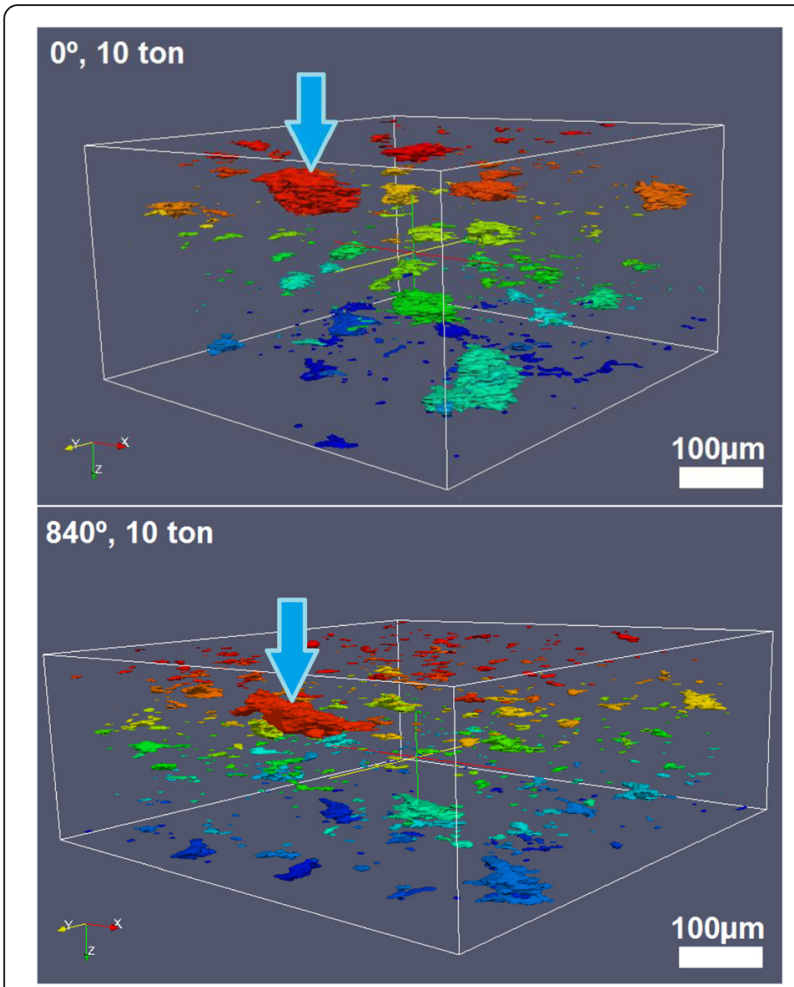

Fig. 11 Evolution of FeS melt distribution in the olivine matrix by shear deformation. Measurements were taken at $1.5 \mathrm{GPa}$ and $1373 \mathrm{~K}$. The San Carlos olivine matrix has been digitally removed to illustrate the 3D distribution of liquid FeS better (colored packets). Sample at $\mathbf{a}-1.5 \mathrm{GPa}$ and $1373 \mathrm{~K}$, before shear deformation and $\mathbf{b}-1.5 \mathrm{GPa}$ and $1373 \mathrm{~K}$, after twisting the upper and lower anvils by $840^{\circ}$. Boxes define the ROls cut out from the entire sample volume. Image courtesy of Heather Watson (personal communications)

\section{Compression mechanism of silica glass: Combining acoustic velocity measurements with HPXMT}

Imaging-based volume/density measurements of noncrystalline materials are intrinsically less accurate, with typical errors on the order of $2 \%$. This is more than ten times the uncertainty in volume/density measurements of crystalline materials based on X-ray diffraction. To characterize equations of state of noncrystalline materials more accurately, Kono et al. (2011) combined HPXMT imaging with ultrasonic velocity measurements. An ultrasonic transducer can be easily mounted on the back side of the PE anvil (e.g., Fig. 4a), without being compressed during high-pressure experiments. Ultrasonic measurements require larger sample diameters in order to obtain strong acoustic signals, and the sample length should also be large enough to avoid overlapping in the acoustic signals reflected at both ends of the sample. In addition, the use of large samples is advantageous for precise measurement of sample volume, although the maximum attainable pressure would be somewhat limited. $\mathrm{A} \mathrm{SiO}_{2}$ glass disk $\sim 2.5 \mathrm{~mm}$ in diameter and $0.429 \mathrm{~mm}$ in length was loaded in the cell assembly. Au foils $(2.5 \mu \mathrm{m}$ thick $)$ were placed at both ends of the sample, serving as boundary/interface markers for length measurements using X-ray imaging. At each pressure point both Pand $\mathrm{S}$-wave travel times were measured at 20 and $30 \mathrm{MHz}$ for S- and P-waves, respectively. X-ray tomography provided direct volume measurements and sample length, which was then used to calculate P- and $\mathrm{S}$-wave velocities from the travel times. By assuming that the glass sample remained elastically isotropic at high pressures, the sample density could be also obtained by integrating the velocities over the pressure range (Kono et al. 2011). This yields a set of density data that is completely independent of sample volume measurements.

Prior to the experiment, the density of the silica disk was determined by Archimedes' method to be $2.208 \mathrm{~g} / \mathrm{cm}^{3}$. This was used to distinguish various thresholding methods in determining the ambient sample volume in HPXMT. It was determined that the Gaussian filter gave the most consistent volume with ambient density data. By adopting the Gaussian filter, relative density changes of the sample at high pressures were determined from volume rendering.

The two density data sets, from ultrasonic velocities and tomographic volume measurements, showed excellent agreement. This allowed further determination of the bulk $(K \mathrm{~s})$ and shear $(G)$ moduli of silica glass. Both $K \mathrm{~s}$ and $G$ decrease with increasing pressure up to $\sim 2.5 \mathrm{GPa}$, above which $K \mathrm{~s}$ and $G$ began to increase again. This softening behavior is related to the polymerized nature of the corner linking $\mathrm{SiO}_{4}$ network in silica, a topic of great interest in the liquid and glass community (e.g., Vukcevich 1972; He and Thorpe 1985). This work demonstrates the power of combining acoustic velocity measurements with HPXMT in studies of the compression behavior of non-crystalline materials. Velocities are directly related to the bulk modulus, which is the pressure derivative of volume. With information on both volume and its pressure derivative, more precise equations of state can be established.

\section{Conclusions}

The HPXMT setup at GSECARS is a powerful in situ imaging tool, serving the high-pressure community with various projects that are of interest to a wide range of scientific disciplines. The system now runs routinely up to $8 \mathrm{GPa}$ in pressure and $2000 \mathrm{~K}$ in temperature. Many improvements and new developments have been undertaken, as described in the paper. On the 10th anniversary of the HPXMT technique, several promising future prospects can be envisioned.

1. Exploring new anvil materials and geometries will allow us to extend the pressure capability significantly. 

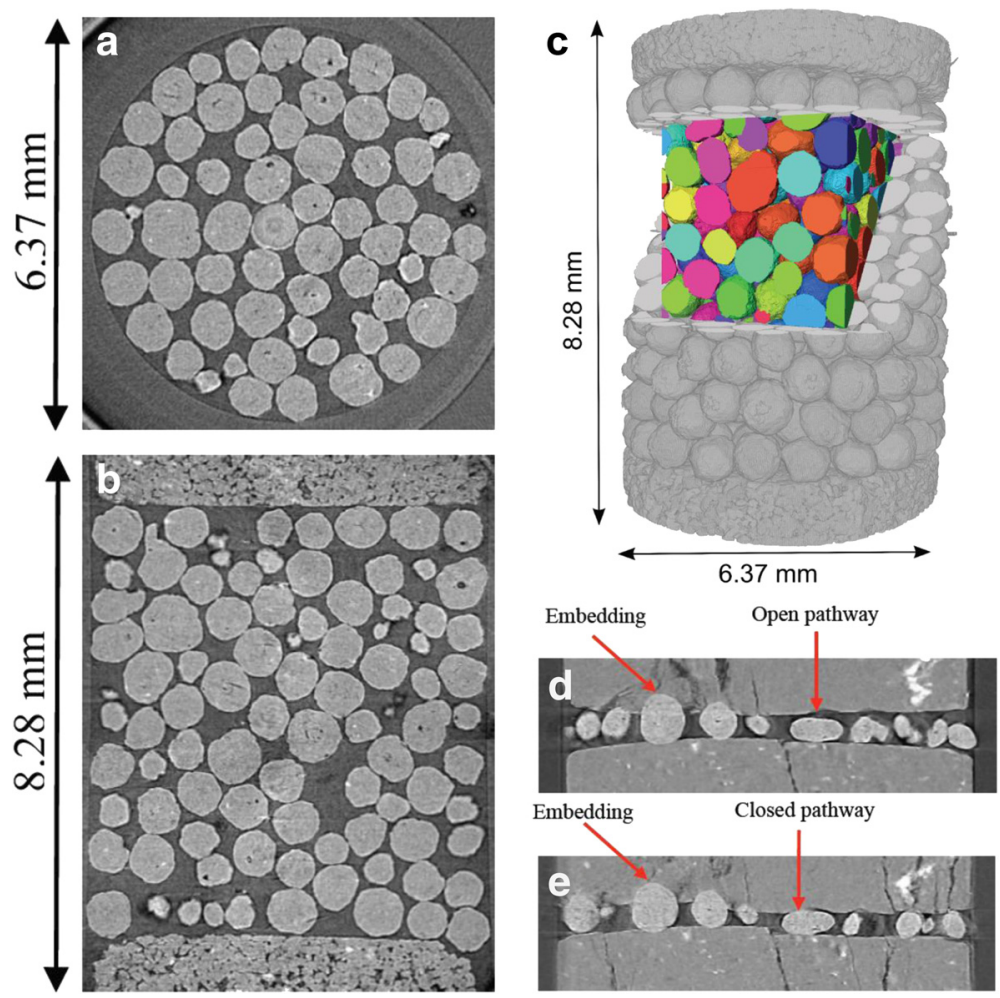

Fig. 12 Imaging the proppant systems with different wall rocks. a Circular cross section of the Berea-proppant sample in the cell shown in Fig. 7a. b Axial cross section, showing both the proppants (circular beads) and the Berea wall rocks (top and bottom slabs). c A cubic ROI within the sample (colored beads) can be digitally selected for solid-void segmentation and detailed permeability analysis. d, e Shale-proppant system simulating a fracture at 0 (d) and $83 \mathrm{MPa}$ (e). The open pathway in $\mathbf{d}$ is closed in $\mathbf{e}$, with significant cracks in the shale wall rock. Images modified after Sanematsu et al. (2015)

The CDT anvils are promising for up to $20 \mathrm{GPa}$. Utilizing sintered diamond as an anvil material will push the pressure limit further. New heater materials will be explored for higher temperature experiments. It may be necessary to upgrade the existing HPXMT apparatus to greater load capacity for higher pressures.

2. For parallel-beam absorption X-ray tomography under pressure, the presence of a pressure medium surrounding the sample presents a challenge for highresolution imaging. Phase contrast tomography can be readily performed in the existing HPXMT apparatus, as the sample-detector distance can be easily adjusted with the integrated nine-axis press/detector manipulation system. This may help improve image quality in some systems. Another option to improve resolution is to combine conventional tomography with known-subregion-based interior tomography (Wang and Yu 2013). Mathematically speaking, tomography produces the reconstruction of a function $F$ from a large number of line integrals of $F$. Conventional tomography is a global procedure because the standard convolution formulae for reconstruction at a given point require the integrals over all lines within the plane containing that point. In contrast, interior construction is a technique to correct truncation artifacts caused by limiting image data to a small field of view. The reconstruction focuses on an area called the region of interest (ROI). The problem of non-uniqueness in interior reconstruction may be solved by global reconstruction at relatively low resolution to facilitate higher resolution interior reconstruction.

3. In addition to absorption tomography, the setup can be readily extended to other $3 \mathrm{D}$ imaging techniques such as X-ray fluorescence tomography of high atomic number elements for chemical composition mapping and diffraction tomography for structural mapping.

4. We have seen an increasing demand for lowpressure (up to several hundred $\mathrm{MPa}$ ) tomography, which requires separate apparatus. We envision the new apparatus to have axial deformation capability, with options for pore fluid and pore pressure control.

\section{Abbreviations}

3D: three-dimensional; APS: Advanced Photon Source; CCD: charge-coupled device; CDT: cupped Drickamer-toroidal; Ce:LuAG: cerium-doped lutetium aluminum garnet; CMOS: complementary metal-oxide-semiconductor; DAC: diamond anvil cell; FEM: finite element method;

GSECARS: GeoSoilEnviroCARS; HPXMT: high-pressure X-ray microtomography; LBF: load-bearing framework; LBM: lattice Boltzmann method; PE: ParisEdinburgh; ROI: region of interest: SPring-8: Super Photon Ring- 8 GeV; YAG: yttrium aluminum garnet. 


\section{Acknowledgements}

We thank T. Uchida for his contribution to the earlier phase of the HPXMT development and F. Westferro for his excellent engineering support. We thank our users for their valuable input, especially C. Lesher, A. Clark, R. Li, Y. Kono, H. Watson, and C. Wilson for their contributions to the science highlights summarized here. The presented experiments were performed at GSECARS (Sector 13), APS, Argonne National Laboratory. APS is supported by the US Department of Energy, Office of Basic Energy Sciences, under Contract No. DE-AC02-06CH11357. GSECARS is supported by the National Science Foundation - Earth Sciences Program (EAR-1128799) and by the Department of Energy - Geosciences (DE-FG02-94ER14466). We are grateful for the generous support of the National Science Foundation (EAR-0001088, 0711057, and 1214376) which made this development possible.

\section{Authors' contributions}

TY and YW set up the experimental hardware and composed the manuscript. MR wrote the tomography data collection, processing, and image reconstruction software. All authors have read and approved the final manuscript.

\section{Competing interests}

The authors declare that they have no competing interests.

\section{Received: 28 November 2015 Accepted: 10 May 2016}

\section{Published online: 08 June 2016}

\section{References}

Alvarez-Murga M, Bleuet P, Marques L, Lepoittvin C, Boudet N, Gabarino G, Mezouar M, Hodeau JL (2011) Microstructural mapping of $C_{60}$ phase transformation into disordered graphite at high pressure using X-ray diffraction microtomography. J Appl Cryst 44:163-171

Balchan A, Drickamer HG (1961) High pressure electrical resistance cell, and calibration points above 100 kbars. Rev Sci Instrum 32:309-313

Bassett WA (2009) Diamond anvil cell, 50th birthday. High Pressure Res 29:163-186

Bromiley GD, Redfern SAT, Le Godec Y, Hamel G, Klotz S (2009) A portable highpressure stress cell based on the V7 Paris-Edinburgh apparatus. High Pressure Res 29:306-316

Clark AN, Lesher CE, Sen S, Jacobsen SD, Wang Y (2013) The physical and elastic properties of silicate melts at high pressure: new insights from high pressure $X$-ray microtomography and gigahertz ultrasonic interferometry, $7^{\text {th }}$ CIDER (Corporative Institute for Dynamic Earth Research) Summer Program. UC Berkeley, Berkeley

Deyhim A, Acome E, Van Every E, Wang Y, Yu T (2012) Development of a nine axis custom design high precision motion system for Advance Photon Source. In: Proceedings of the $7^{\text {th }}$ International Conference on Mechanical Engineering Design of Synchrotron Radiation Equipment and Instrumentation, Shanghai, China, 15-19 Oct

He H, Thorpe MF (1985) Elastic properties of glasses. Phys Rev Lett 54:2107-2110

Ketcham RA (2005) Computational methods for quantitative analysis of threedimensional features in geological specimens. Geosphere 1:32-41

Khvostantsev LG, Vereshchagin LF, Novikov AP (1977) Device of toroid type for high pressure generation. High Temp-High Press 9:637-639

Kleine T, Touboul M, Bourdon B, Nimmo F, Mezger K, Palme H, Halliday AN (2009) Hf-W chronology of the accretion and early evolution of asteroids and terrestrial planets. Geochem Cosmochim AC 73(17):5150-5188

Kono Y, Yamada A, Wang Y, Yu T, Inoue T (2011) Combined ultrasonic elastic wave velocity and microtomography measurements at high pressures. Rev Sci Instrum 82:023906

Kono Y, Park C, Kenney-Benson C, Shen G, Wang Y (2014) Toward comprehensive studies of liquids at high pressures and high temperatures: combined structure, elastic wave velocity, and viscosity measurements in the ParisEdinburgh cell. Phys Earth Planet In 228:269-280

Lesher CE, Wang Y, Gaudio S, Clark A, Nishiyama N, Rivers M (2009) Volumetric properties of magnesium silicate glasses and supercooled liquid at high pressure by X-ray microtomography. Phys Earth Planet In 174(1-4):292-301

Li R, Li L, Yu T, Wang L, Chen J, Wang Y, Cai Z, Chen J, Rivers ML, Liu H (2014) Study of liquid gallium as a function of pressure and temperature using synchrotron X-ray microtomography and X-ray diffraction. Appl Phys Lett 105:041906
Liebermann RC (2011) Multi-anvil, high pressure apparatus: a half-century of development and progress. High Pressure Res 31:493-532

Liu H, Wang L, Xiao X, De Carlo F, Feng J, Mao HK, Hemley RJ (2008) Anomalous high-pressure behavior of amorphous selenium from synchrotron X-ray diffraction and microtomography. Proc Natl Acad Sci 105:13229

Morard G, Mezouar M, Rey N, Poloni R, Merlen A, Le Floch S, Toulemonde P, Pascarelli S, San-Miguel A, Sanloup C, Fiquet G (2007) Optimization of ParisEdinburgh press cell assemblies for in situ monochromatic $X$-ray diffraction and X-ray absorption. High Pressure Res 27:223-233

Rivers ML (1998) Tutorial introduction to X-ray computed microtomography data processing. http://www.mcs.anl.gov/research/projects/X-ray-cmt/rivers/ tutorial.html. Accessed 14 May 1998

Rivers ML (2012) tomoRecon: high-speed tomography reconstruction on workstations using multi-threading. In: Stock SR (ed) Proceedings of SPIE, Developments in X-Ray Tomography VIII, 8506., p 85060U

Rivers ML, Sutton SR, Eng P (1999) Geoscience applications of X-ray computed microtomography. In: Bonse U (ed) Proceedings of SPIE, Developments in X-Ray Tomography II 3772., pp 78-86

Rivers ML, Citron DT, Wang Y (2010) Recent developments in computed tomography at GSECARS. In: Stock SR (ed) Proceedings of SPIE, Developments in X-Ray Tomography VII, 7804., p 780409

Rotenberg Y, Boruvka L, Neumann AW (1983) Determination of surface tension and contact angle from the shapes of axisymmetric fluid interfaces. J Colloid Interf Sci 93:169-183

Sanematsu P, Shen Y, Thompson K, Yu T, Wang Y, Chang D, Alramahi B, TakbiriBorujeni A, Tyagi M, Wilson C (2015) Image-based Stokes flow modeling in bulk proppant packs and propped fractures under high loading stresses. J Petrol Sci Eng 135:391-402

Terasaki H, Urakawa S, Funakoshi K, Wang Y, Shibazaki Y, Sanehira T, Ueda Y, Ohtani E (2008) Interfacial tension measurement of Ni-S liquid using highpressure X-ray micro-tomography. High Pressure Res 28(3):327-334

Terasaki H, Urakawa S, Funakoshi K, Nishiyama N, Wang Y, Nishida K, Sakamaki T, Suzuki A, Ohtani E (2009) In situ measurement of interfacial tension of Fe-S and Fe-P liquids under high pressure using $X$-ray radiography and tomography techniques. Phys Earth Planet In 174:220-226

Todd KA (2013) The effects of shear deformation on planetesimal core segregation: results from in-situ X-ray microtomography. Thesis. Northern Illinois University

Todd KA, Watson HC, Yu T, Wang Y (2016) The effects of shear deformation on planetesimal core segregation: results from in-situ X-ray microtomography. Am Mineral (accepted)

Urakawa S, Terasaki H, Funakoshi K, Uesugi K, Yamamoto S (2010) Development of high pressure apparatus for X-ray microtomography at SPring-8. J Phys Conf Ser 215:012026

Vukcevich MR (1972) A new interpretation of the anomalous properties of vitreous silica. J Non Cryst Solids 11:25-63

Wang G, Yu H (2013) The meaning of interior tomography. Phys Med Biol 58(16):R161-186

Wang Y, Uchida T, Westferro F, Rivers ML, Nishiyama N, Gebhardt J, Lesher CE, Sutton SR (2005) High-pressure X-ray tomography microscope: synchrotron computed microtomography at high pressure and temperature. Rev Sci Instrum 76:073709

Wang Y, Rivers ML, Sutton SR, Nishiyama N, Uchida T, Sanehira T (2009) The large-volume high-pressure facility at GSECARS: a "Swiss-army-knife" approach to synchrotron-based experimental studies. Phys Earth Planet In 174:270-281

Wang Y, Lesher C, Fiquet G, Rivers ML, Nishiyama N, Siebert J, Roberts J, Morard G, Gaudio S, Clark A, Watson H, Menguy N, Guyot F (2011) In situ highpressure and high-temperature $\mathrm{X}$-ray microtomographic imaging during large deformation: a new technique for studying mechanical behavior of multiphase composites. Geosphere 7(1):40-53

Wilding MC, Benmore CJ, Tangeman JA, Sampath S (2004) Coordination changes in magnesium silicate glasses. Europhys Lett 67:212-218 